\title{
Twisted Edwards Elliptic Curves for Zero-Knowledge Circuits
}

\author{
Marta Bellés-Muñoz ${ }^{1, *(\mathbb{D})}$, Barry Whitehat ${ }^{2}$, Jordi Baylina ${ }^{3}$, Vanesa Daza ${ }^{1}$ (D) and Jose Luis Muñoz-Tapia ${ }^{4}$ (D) \\ 1 Department of Information and Communications Technology, Pompeu Fabra University, Tànger Building, \\ 08018 Barcelona, Spain; vanesa.daza@upf.edu \\ 2 Independent Researcher, 6300 Zug, Switzerland; barrywhitehat@protonmail.com \\ 3 0KIMS, Eschenring 11, 6300 Zug, Switzerland; jordi@baylina.cat \\ 4 Department of Network Engineering, Campus Nord, Polytechnic University of Catalonia, \\ 08034 Barcelona, Spain; jose.luis.munoz@upc.edu \\ * Correspondence: marta.belles@upf.edu
}

Citation: Bellés-Muñoz, M.; Whitehat, B; Baylina, J.; Daza, V.; Muñoz-Tapia, J.L. Twisted Edwards Elliptic Curves for Zero-Knowledge Circuits. Mathematics 2021, 9, 3022. https://doi.org/10.3390/ math9233022

Academic Editors: Ioana Boureanu and Liqun Chen

Received: 31 October 2021

Accepted: 19 November 2021

Published: 25 November 2021

Publisher's Note: MDPI stays neutral with regard to jurisdictional claims in published maps and institutional affiliations.

Copyright: (c) 2021 by the authors. Licensee MDPI, Basel, Switzerland. This article is an open access article distributed under the terms and conditions of the Creative Commons Attribution (CC BY) license (https:// creativecommons.org/licenses/by/ $4.0 /)$.

\begin{abstract}
Circuit-based zero-knowledge proofs have arose as a solution to the implementation of privacy in blockchain applications, and to current scalability problems that blockchains suffer from. The most efficient circuit-based zero-knowledge proofs use a pairing-friendly elliptic curve to generate and validate proofs. In particular, the circuits are built connecting wires that carry elements from a large prime field, whose order is determined by the number of elements of the pairing-friendly elliptic curve. In this context, it is important to generate an inner curve using this field, because it allows to create circuits that can verify public-key cryptography primitives, such as digital signatures and encryption schemes. To this purpose, in this article, we present a deterministic algorithm for generating twisted Edwards elliptic curves defined over a given prime field. We also provide an algorithm for checking the resilience of this type of curve against most common security attacks. Additionally, we use our algorithms to generate Baby Jubjub, a curve that can be used to implement elliptic-curve cryptography in circuits that can be validated in the Ethereum blockchain.
\end{abstract}

Keywords: zero-knowledge proof; elliptic curve; blockchain; privacy

\section{Introduction}

Since the first occurrence of an elliptic curve in one of Diophantus' Arithmetica books, elliptic curves have played an increasingly important role in mathematics. These mathematical objects showed their practical potential in the 1980s, when Koblitz [1] and Miller [2] independently proved that some techniques used in modern cryptography could also be applied to elliptic curve groups, and that the resulting schemes were more efficient. After four decades of research and development, elliptic-curve cryptography (ECC) now has widespread exposure and acceptance; and industry, banking, and government standards, have already migrated from classic public-key cryptography to ECC [3].

In 2008, blockchain, the technology behind most cryptocurrencies, was added to the list of practical uses for ECC. In Satoshi's seminal Bitcoin paper [4], ECC was used for securing the various transactions occurring on the network, for controlling the generation of new currency units, and verifying the transfer of digital assets and tokens. A few years later, privacy-oriented cryptocurrencies, such as Monero and Zcash, incorporated new cryptographic techniques to ensure user anonymity and obfuscate payment details. For instance, Monero started using ring signatures and Pedersen commitments [5], while Zcash exploited a type of protocols called zero-knowledge (ZK) proofs [6].

$\mathrm{ZK}$ proofs allow one party to convince another that a statement is true without revealing any information beyond the veracity of the statement [7]. In general, they are used to show that someone knows the result of a computation without revealing the solution. For example, with ZK proofs, one can prove that someone holds the private key associated with a certain public key without revealing the private key. Another example is 
to prove that a transaction has been computed correctly without leaking any information about the transaction details.

Among different ZK systems, the most suitable for blockchain applications are the ones called ZK succinct non-interactive arguments of knowledge (ZK-SNARKs). This type of protocol does not require any interaction between the parties involved, and the size of the proofs is small [8-10]. Most ZK-SNARK constructions make use of bilinear pairings over elliptic curve groups to achieve efficient verification, too. In this way, blockchain users can send proofs as part of a transaction to a smart contract, who verifies if the proof is correct and performs a specific action depending on whether the proof verification is satisfied or not.

Before proving computational statements with ZK-SNARKs, statements have to be expressed as an $\mathbb{F}_{p}$-arithmetic circuit (also called ZK circuit or ZK-SNARK circuit), which is a circuit made up of additions and multiplications modulo a certain prime $p$. The specific construction of the protocol is what determines $p$, but typically $p$ is a large prime number of approximately 254 bits that is determined by the order of a pairing-friendly elliptic curve [11]. For instance, in Ethereum, $p$ is the order of BN256 elliptic curve, and in Zcash, $p$ is the large prime order subgroup of BLS12-381.

Classical cryptographic schemes consisted mostly of boolean operations, which makes them inefficient when evaluated inside a ZK-SNARK circuit. As an example, the Zcash circuit relied on the SHA256 hash function to create a message-authentication code to prevent malleability, for generating pseudo-random strings and for commitments. However, each invocation of SHA256 added tens of thousands of multiplication gates to ZK-SNARK circuits, making this hash the primary cost when generating ZK-SNARK proofs [12]. These issues motivated the search for algebraic primitives to replace SHA256 and other inefficient functions. So, instead of hash functions such as SHA256 inside ZK-SNARK circuits, the idea was to use ECC that works in large prime fields, which is the natural representation of circuits. For this reason, new schemes that relied on elliptic curves were gradually adopted in ZK-SNARK circuits.

In this context, two prominent examples are Pedersen hashes [13] and the Edwards digital signature algorithm (EdDSA) [14]. These schemes can be built efficiently by using elliptic curves that can be represented in the twisted Edwards form.The nice feature of this form is that there is a single formula for doubling and adding points of the curve (Section 6, [15]). There is another form of representing elliptic curves called Montgomery, that makes computations faster but has different formulas for adding and doubling points [16]. The nice thing is that the twisted Edwards form is generally birationally equivalent to a Montgomery curve, so the curve can be easily converted from one form to another (Theorem 3.2, [15]). Inside our ZK-SNARK circuit, we can use the Montgomery form when we know for sure that either we are adding different points or we are adding the same point, and use twisted Edwards when, depending on the inputs of the circuit, this cannot be assured. Combining the two forms in this way makes the implementation of the group law inside ZK-SNARK circuits very efficient.

\subsection{Motivation}

In order to implement the Pedersen hash and the EdDSA inside a ZK-SNARK circuit, one needs curves that are defined over the finite field of prime order $p$, namely $\mathbb{F}_{p}$, where $p$ is determined by the particular choice of pairing-friendly elliptic curve used to generate ZK-SNARK proofs. It is crucial to choose an appropriate twisted Edwards elliptic curve with optimal parameters for the cryptographic schemes, since the choice of the curve has great impact on their security and efficiency. Moreover, it is important to generate curves in a transparent and deterministic way, so that anyone can audit and recreate the procedure. Transparency is paramount, as it significantly reduces the possibility of a backdoor being present, thus leading to better security. Needless to say, it is crucial that the new curves are also tested for resilience against best known attacks, such as the rho method, or additive 
and multiplicative transfers, which attack the discrete logarithm problem over elliptic curve groups [17].

\subsection{Our Contributions}

In this paper, we present a set of deterministic algorithms that, given a field $\mathbb{F}_{p}$, allows us to generate secure twisted Edwards elliptic curves that are suitable for $\mathbb{F}_{p}$-arithmetic circuits and allow the efficient computation of ZK-SNARK proofs that prove ECC statements. There have already been two curves that have been generated using these methods. On the one side, Jubjub curve for Zcash, defined over the scalar field of BLS12-381 and on the other side, Baby Jubjub for Ethereum, defined over the scalar field of BN256. The present work is a formalization and generalization of the common efforts to generate suitable twisted Edwards curves for ZK-SNARK circuits.

\subsection{Organization of the Paper}

The rest of the paper is structured in five main sections. In Section 2, we introduce related work on generation of elliptic curves in the ZK context. In the following Section 3, we provide a general overview of ZK proofs and classical theory of elliptic curves. In Section 4, we present a deterministic algorithm for generating twisted Edwards elliptic curves defined over a given prime field. We complement the work in Section 5, with the security checks that a twisted Edwards elliptic curve should pass. We base this section on the work from Bernstein and Lange gathered in [18]. In Section 6, we use our algorithms to generate Baby Jubjub, a curve that can be used to implement elliptic-curve schemes inside $\mathbb{F}_{p}$-arithmetic circuits for Ethereum, and which has already been used in practical applications such as Hermez, a Layer-2 payment system, and Tornado Cash, a payment mixer. Finally, we conclude in Section 7 with some discussion of the work and future research directions. Additionally, we provide Appendix A with a SAGE implementation of the security checks presented in Section 5, which was used to prove that Baby Jubjub was safe under best known security attacks.

\section{Related Work}

The interest of society and regulators in privacy, both individual's privacy and industry trade secrets, has grown in the recent years, and has ended up with new legislation such as the General Data Protection Regulation (GDPR) passed by the European Union [19] and the California Consumer Privacy Act (CCPA) [20]. Privacy is specially relevant in blockchain, where it is crucial to combine the inherent transparency of the system with the users privacy rights [21-23]. To this purpose, in the blockchain space, there has been an intensive use of cryptographic schemes that make use of elliptic curves, which has motivated the appearance of new problems that have been tackled by both industry and academia.

For instance, the need for efficient pairing-friendly curves in ZK-SNARK schemes resurfaced the work from Barreto and Naehrig [24], and Barreto, Lynn, and Scott [25], who developed techniques to generate pairing-friendly elliptic curves that had an optimal Ate pairing. The vast application of curves derived from their work, which are usually called BN and BLS curves, resulted in undergoing processes from the IRTF Crypto Forum Research Group to standardize particular instantiations of these curves [26,27], such as the BN-256 and the BLS12-381, which are used in digital signature schemes and ZK-SNARK protocols all over the Internet.

The order of these curves is what determines the type of statements we can prove using ZK. More precisely, the largest prime $p$ dividing the curve's order fixes the field in which we can do modular arithmetic. As a result, computational statements that involve elliptic-curve operations can only be proved efficiently with curves that are defined over the prime field $\mathbb{F}_{p}$. Hence, the implementation of ECC schemes that make use of twisted Edwards curves require new curves defined over $\mathbb{F}_{p}$. Table 1 summarizes the most closely related contributions to our work. 
Table 1. Comparison with related work.

\begin{tabular}{ccc}
\hline System & Outer Curve & Inner Curve \\
\hline Zcash [12] & BLS12-381 & Jubjub \\
Masson et al. [28] & BLS12-381 & Bandersnatch \\
Our Proposal & BN-256 & Baby Jubjub \\
Ben-Sasson et al.[29] & MNT4 & MNT6 \\
ZEXE [30] & CP6-782 & BLS12-377 \\
Housni et al. [31] & BW6-761 & BLS12-377 \\
\hline
\end{tabular}

The Zcash team was the first to generate a suitable curve for $\mathbb{F}_{p}$-arithmetic circuits. Since Zcash ZK-SNARK constructions are based on BLS12-381, their curve, named Jubjub, was expressly built over the BLS12-381 scalar field.

Recently, a new elliptic curve built over the BLS12-381 scalar field was introduced in [28], but although this curve allows a faster scalar multiplication algorithm than Jubjub, it does not provide any performance improvement in multi-scalar multiplications or in the ZK circuit representations.

We have used a similar approach to generate Baby Jubjub, which is an embedded elliptic curve designed to operate on the field produced by the BN256 elliptic curve. This work that we are presenting covers the case in which we want to use circuits that can verify public key cryptography primitives such as digital signatures and encryptions in Ethereum. Then, these proofs can be verified by an Ethereum smart contract because the Ethereum virtual machine (EVM) has an operation to compute pairings with the BN256 elliptic curve. We presented and discussed the techniques used to generate Jubjub and Baby Jubjub at the second annual ZKProof Standardization Workshop in Berkeley. The present work is a reviewed, formalized and generalized version of the efforts towards the standardization of the generation of suitable twisted Edwards curves for ZK-SNARK circuits.

The generation of other type of curves for ZK-SNARK circuits has also appeared in other lines of research. For instance, the authors of [29] presented the first practical setting of recursive proof composition with a cycle of two Miyaji-Nakabayashi-Takano (MNT) pairing-friendly elliptic curves [32]. The idea of their proposal is that proofs generated from one curve can feasibly reason about proofs generated from the other curve. To achieve this, one curve's order is the other curve's base field order and vice versa. Although current MNT cycles of curves are quite expensive at the 128-bit security, the work opens the door to the possibility of having succinct blockchains that are verifiable with one single proof. Bowe et al. [30] proposed ZEXE, a construction that follows a relatively relaxed approach to find a suitable pair of curves that form a chain rather than a cycle. A later work from El Housni and Guillevic [31] improved ZEXE with a new curve that makes the verification of composed ZK-SNARK proofs significantly faster.

\section{Background}

In this section, we review the main ideas behind $\mathrm{ZK}$ proving systems and elliptic curve theory, focusing on twisted Edwards and Montgomery forms.

\subsection{Zero-Knowledge Proofs}

Zero-knowledge (ZK) proofs allow one party, called prover, to convince another one, called verifier, that a statement is true without revealing any information beyond the veracity of the statement. In this context, we understand a statement as a relation between an instance, a public input known to both prover and verifier, and a witness, a private input only known by the prover, which belongs to a language $\mathcal{L}$ in the nondeterministic polynomial time (NP) complexity class [7]. More specifically, a ZK protocol satisfies that a verifier will accept the proof if it is generated by an honest prover (completeness) and the verifier will not accept proofs from a dishonest prover (soundness). These two properties protect the verifier against dishonest provers. On the other hand, ZK proofs 
must ensure that the proofs do not leak any information about the statement being proved (zero-knowledge), guaranteeing this way the privacy of the prover's secret information against malicious verifiers [7].

Most ZK systems operate in the model of $\mathbb{F}_{p}$-arithmetic circuits, which are circuits composed by wires that carry values from a prime field $\mathbb{F}_{p}$ and connect them to addition and multiplication gates modulo $p$. We consider that an assignment to the wires is valid if for every gate, the value on the output wires matches that gate's operation and the values on its input wires. In this context, a ZK proof typically allows to prove the existence of a valid circuit wires assignment, but there is a special class of protocols known as arguments of knowledge that allow to prove that, with very high probability, the prover knows a valid assignment to the wires of the circuit.

An argument of knowledge is considered a ZK succinct non-interactive argument of knowledge (ZK-SNARK) if there is no interaction between the prover and the verifier during the generation of a proof and, regardless of the size of the statement being proved, has succinct proof size (e.g., [9]—proofs are $\approx 200$ bytes). Most ZK-SNARKs also guarantee short verification time by making use of bilinear pairings over groups of points of an elliptic curve [8-10]. The largest prime $p$ diving of the curve is what determines the modular arithmetic performed in the arithmetic circuits. For instance, a ZK-SNARK protocol that uses pairings over an elliptic curve of prime order $p$ allows proving statements regarding $\mathbb{F}_{p}$-arithmetic circuits.

\subsection{Elliptic Curves}

In this section, we first give a general overview of elliptic curves defined over any field $K$, following the definitions and notation in [33] (Chapter VI), and then, we focus on twisted Edwards and Montgomery elliptic curves defined over prime finite fields. For general results about elliptic curves, we refer the reader to $[34,35]$.

Definition 1 (Elliptic curve). Let $K$ be a field of characteristic $\neq 2,3$ and let

$$
y^{2}+a_{1} x y+a_{3} y=x^{3}+a_{2} x^{2}+a_{4} x+a_{6}
$$

be a cubic polynomial equation with $a_{1}, \ldots, a_{6} \in K$ and with no multiple roots. An elliptic curve $E$ over $K$ consists of the set of points $(x, y)$ with $x, y \in K$ which satisfy Equation (1), together with a single element called point at infinity, denoted by $\mathcal{O}$.

An important result about the set of points of an elliptic curve is that they form an additive Abelian group. To define the addition of points, which might seem a little unnatural at first, it is easier to think of elliptic curves as objects in the two-dimensional projective space.

Group Law 1. Let $E$ be an elliptic curve and $P, Q$ points on $E$. Let $L \subset \mathbb{P}^{2}$, where $\mathbb{P}^{2}$ denotes the 2-dimensional projective space, be the line that intersects both $P$ and $Q$ and denote by $R$ the third point of intersection between $E$ and $L$. Let $L^{\prime}$ be the line that intersects both $R$ and $\mathcal{O}$. We define $P+Q$ as the third point of intersection between $E$ and $L^{\prime}$.

The following example illustrates visually the composition of points in the plane of an ordinary curve defined over the field of real numbers $\mathbb{R}$.

Example 1. Let $E$ be the elliptic curve $E: y^{2}=x^{3}-2 x$ defined over $\mathbb{R}$. In Figure 1 , we see the composition $P+Q$ for two points $P$ and $Q$ with distinct $y$-coordinates. The line through the points intersects the curve in a third point $R$ and $P+Q$ is the reflection across the $x$-axis. 


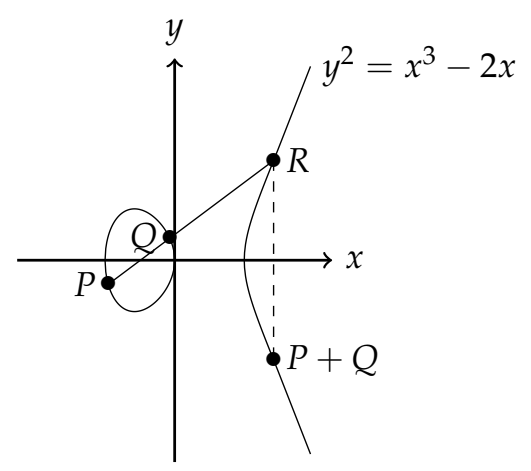

Figure 1. The Group Law for two distinct points $P, Q$ on the elliptic curve $E: y^{2}=x^{3}-2 x$.

Proposition 1. The composition of points on an elliptic curve E defined in Group Law 1 makes $E$ into an additive Abelian group with identity $\mathcal{O}$.

Proof. See (Proposition 2.2, Chapter III, [34]).

In cryptography, we are interested in elliptic curves over finite fields. For the rest of the article, we shall let $K$ be a prime-order finite field $\mathbb{F}_{p}$ for some prime $p$. In this context, it makes sense to define the order of the curve and the order of a point.

Definition 2 (Order). The order of an elliptic curve defined over $\mathbb{F}_{p}$ is its number of points. The order of a point $P$ is the smallest positive integer $n$ such that $n P=\mathcal{O}$.

The following result is a particular case of Hasse's theorem, which provides an upper and a lower bound of the order of an elliptic curve.

Theorem 1 (Hasse's Bound Theorem). Let $n$ be the number of points on an elliptic curve defined over $\mathbb{F}_{p}$. Then,

$$
|n-(p+1)| \leq 2 \sqrt{p}
$$

Proof. See (Theorem 1.1, Chapter V, [34]).

Definition 3. Let $n$ be the order of an elliptic curve $E$. We say that a point $G_{0}$ is a generator of the curve, if it has order $n$. When $n$ is a composite number of the form $n=h \times l$, where $h$ is a small number (typically called cofactor) and $l$ is a large prime number, we say that $G_{1}$ is a base point, if it has order $l$.

\section{Montgomery and Twisted Edwards Curves}

Below, we define and describe elliptic curves in Montgomery and twisted Edwards form. In this part, we follow $[15,36]$.

Definition 4 (Montgomery curve). Let $p \geq 3$ be a prime and $\mathbb{F}_{p}$ the finite field of order $p$. For $A, B \in \mathbb{F}_{p}, A \in \mathbb{F}_{p} \backslash\{-2,2\}$ and $B \in \mathbb{F}_{p} \backslash\{0\}$, an elliptic curve defined by

$$
E^{M}: B y^{2}=x^{3}+A x^{2}+x
$$

is called a Montgomery (elliptic) curve.

The following theorem presents the addition formulas for Montgomery curves.

Theorem 2. Let $P_{1}=\left(x_{1}, y_{1}\right) \neq \mathcal{O}$ and $P_{2}=\left(x_{2}, y_{2}\right) \neq \mathcal{O}$ be two points of a Montgomery curve $E^{M}$. Then: 
- If $P_{1} \neq P_{2}$, then the sum $P_{1}+P_{2}$ is a third point $P_{3}=\left(x_{3}, y_{3}\right)$ with coordinates

$$
\begin{aligned}
& \Lambda=\left(y_{2}-y_{1}\right) /\left(x_{2}-x_{1}\right), \\
& x_{3}=B \Lambda^{2}-A-x_{1}-x_{2}, \\
& y_{3}=\Lambda\left(x_{1}-x_{3}\right)-y_{1} .
\end{aligned}
$$

- If $P_{1}=P_{2}$, then $P_{1}+P_{1}$ is a point $P_{3}=\left(x_{3}, y_{3}\right)$ with coordinates

$$
\begin{aligned}
& \Lambda=\left(3 x_{1}^{2}+2 A x_{1}+1\right) /\left(2 B y_{1}\right), \\
& x_{3}=B \Lambda^{2}-A-2 x_{1}, \\
& y_{3}=\Lambda\left(x_{1}-x_{3}\right)-y_{1} .
\end{aligned}
$$

Proof. See [16].

Theorem 3. The order of a Montgomery curve is divisible by 4.

Proof. See (Section 10.3.2, [36]).

Definition 5 (Twisted Edwards curve). Let $p \geq 3$ be a prime and $\mathbb{F}_{p}$ the finite field of order $p$. For distinct $a, b \in \mathbb{F}_{p} \backslash\{0\}$, an elliptic curve defined by

$$
E: a x^{2}+y^{2}=1+d x^{2} y^{2}
$$

is called a twisted Edwards (elliptic) curve.

As the next theorem shows, twisted Edwards curves have complete addition formulas, which makes these curves very efficient to implement inside ZK-SNARK circuits.

Theorem 4. Let $P_{1}=\left(x_{1}, y_{1}\right)$ and $P_{2}=\left(x_{2}, y_{2}\right)$ be points of a twisted Edwards elliptic curve E. The sum $P_{1}+P_{2}$ is a third point $P_{3}=\left(x_{3}, y_{3}\right)$ with

$$
\begin{aligned}
& \lambda=d x_{1} x_{2} y_{1} y_{2}, \\
& x_{3}=\left(x_{1} y_{2}+y_{1} x_{2}\right) /(1+\lambda), \\
& y_{3}=\left(y_{1} y_{2}-x_{1} x_{2}\right) /(1-\lambda) .
\end{aligned}
$$

Note that the inverse of a point $(x, y)$ in a twisted Edwards curve is $(-x, y)$.

Proof. See ([37], Section 3).

The following theorem states that Montgomery and twisted Edwards curves are birationally equivalent. The theorem also gives the birational map that allows the transformation from one form to the other.

Theorem 5. Every twisted Edwards curve E over $\mathbb{F}_{p}$ is birationally equivalent over $\mathbb{F}_{p}$ to a Montgomery curve $E^{M}$ with parameters

$$
A=2 \frac{a+d}{a-d} \quad \text { and } \quad B=\frac{4}{a-d} .
$$

The birational equivalence from $E$ to $E^{M}$ is the map

$$
(x, y) \rightarrow(u, v)=\left(\frac{1+y}{1-y}, \frac{1+y}{(1-y) x}\right)
$$


with inverse

$$
(u, v) \rightarrow(x, y)=\left(\frac{u}{v}, \frac{u-1}{u+1}\right) .
$$

Conversely, every Montgomery curve over $\mathbb{F}_{p}$ is birationally equivalent over $\mathbb{F}_{p}$ to a twisted Edwards curve with parameters

$$
a=\frac{A+2}{B} \text { and } \quad d=\frac{A-2}{B} .
$$

Proof. See (Theorem 3.2, [15]).

\section{Our Proposal}

In this section, we present a method that, given a prime number $p$, we get a twisted Edwards curve defined over $\mathbb{F}_{p}$.

\subsection{General Overview}

Our algorithm takes a prime number $p$ and returns a twisted Edwards curve defined over $\mathbb{F}_{p}$. More precisely, the specific outputs of the algorithm are:

- The prime order of the finite field the curve is defined over (which is the input $p$ );

- $\quad$ Parameters $a$ and $d$ of the equation that defines the twisted Edwards curve;

- The order of the curve and its decomposition into the product of a cofactor and a large prime;

- $\quad$ A generator and a base point for the curve.

Since the finite field is defined by the input $p$, no specification of this parameter is required. The order of the curve and its decomposition are also determined once the parameters of the equation describing the curve are fixed. Hence, the only remaining specifications are parameters $a$ and $d$ and the choice of generator and base point.

We have divided the procedure in four steps:

1. Choice of Montgomery Equation: we start by deterministically generating a Montgomery elliptic curve $E^{M}$ over $\mathbb{F}_{p}$;

2. Choice of Generator and Base Points: we set the generator and base points of $E^{M}$;

3. Transformation to Twisted Edwards: we convert the curve $E^{M}$ to its birationally equivalent twisted Edwards form and the generator and base points using the maps from Theorem 5;

4. Optimization of Parameters: if possible, we rescale all parameters so that the arithmetic in the curve can be sped up [38].

All algorithms presented in this section have been implemented in SAGE programming language, and are presented in Section 6.

\subsection{Choice of Montgomery Equation}

We start by finding a Montgomery curve defined over $\mathbb{F}_{p}$ where $p$ is a given prime number. The assumptions and algorithm presented are based on the work of [39] and Zcash team [40].

Algorithm 1 takes a prime $p$, fixes $B=1$ and returns the Montgomery elliptic curve defined over $\mathbb{F}_{p}$ with the smallest coefficient $A$ such that $A-2$ is a multiple of 4 . This approach comes from the fact that, when defining a Montgomery curve, the smaller $A$ is, the faster the group operation becomes. More precisely, as pointed out in [41], for the best performance, we need $(A-2) / 4$ to be small. As with $A=1$ and $A=2$, a twisted Edwards equation does not describe a smooth curve, so the algorithm starts with $A=3$.

For primes congruent to 1 modulo 4 , the minimal cofactors of the curve and its twist are either $\{4,8\}$ or $\{8,4\}$. We choose a curve with the latter cofactors, so that any algorithms that take the cofactor into account do not have to worry about checking for points on the 
twist, because the twist cofactor will be the smaller of the two [39]. For a prime congruent to 3 modulo 4 , both the curve and twist cofactors can be 4 , and this is minimal.

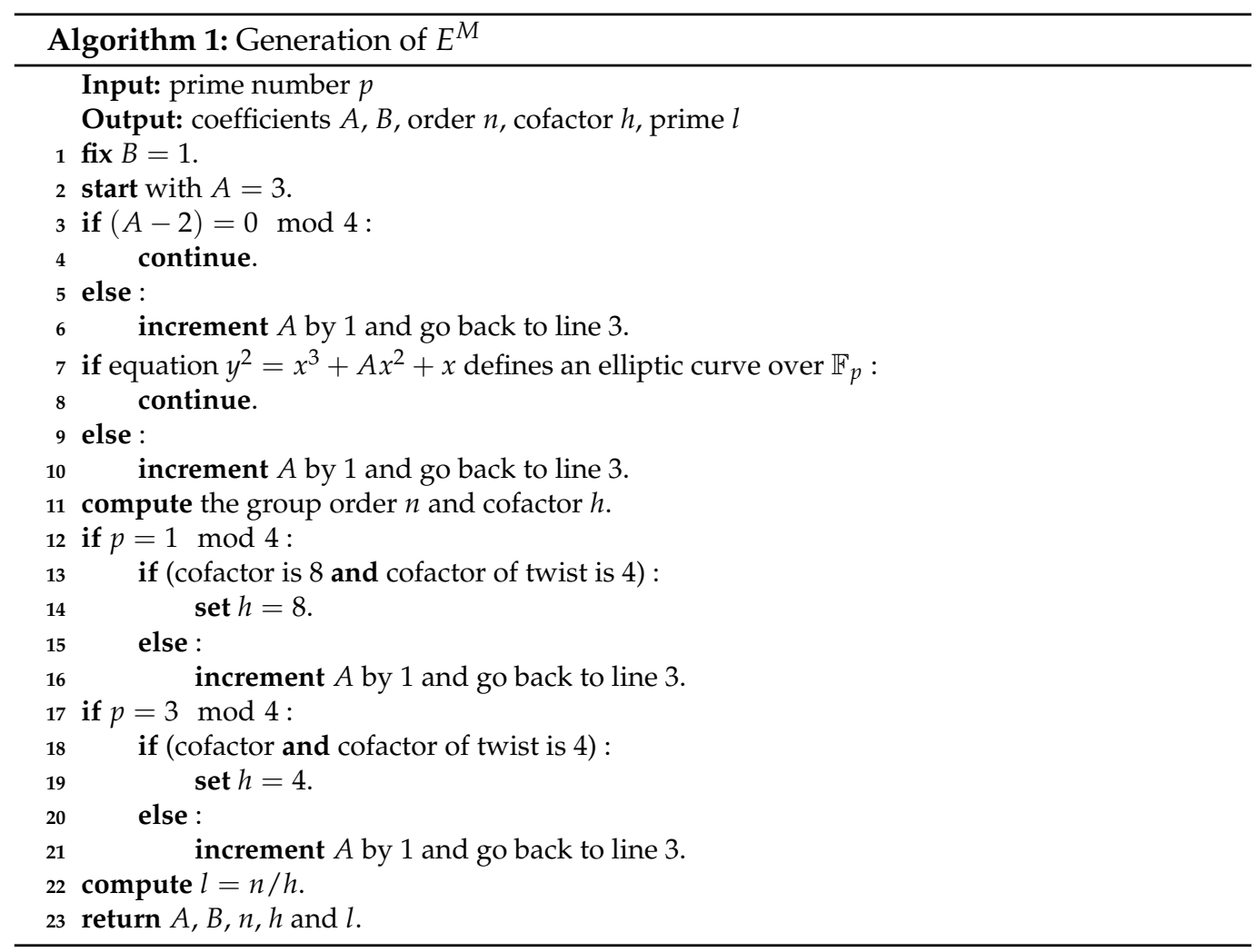

\subsection{Choice of Generator and Base Points}

To pick a generator $G_{0}^{M}$ of the curve, we choose the smallest element of $\mathbb{F}_{p}$ that corresponds to an $x$-coordinate of a point in the curve of order $n$. Then, as a base point, we define $G_{1}^{M}=8 \cdot G_{0}^{M}$, which has order $l$. The steps are written down in Algorithm 2.

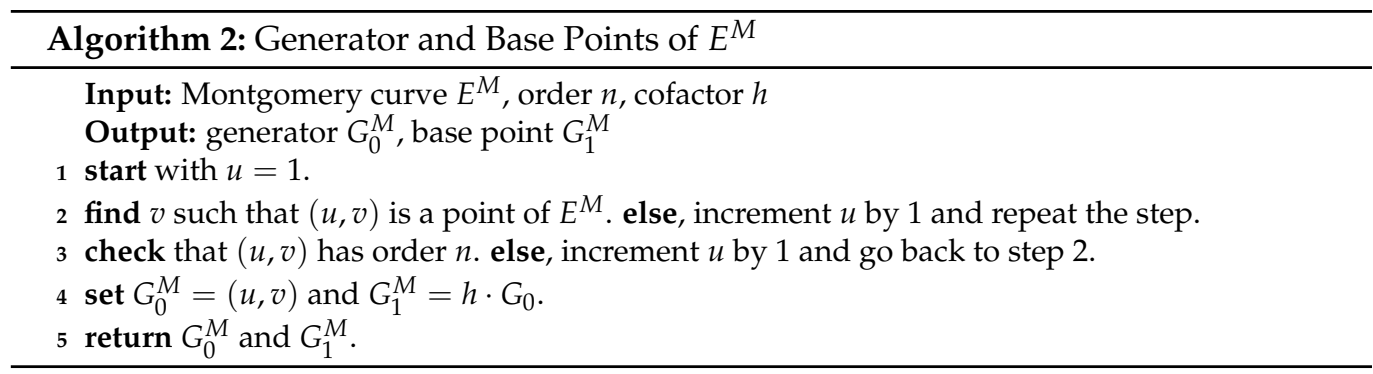

\subsection{Transformation to Twisted Edwards}

In Algorithm 3, we use the birational map from Equation (4) to get the coefficients, generator and base points in the twisted Edwards form.

\subsection{Optimization of Parameters}

As pointed out in [38] (Section 3.1), if $-a$ is a square in $\mathbb{F}_{p}$, it is possible to optimize the number of operations in a twisted Edwards curve by scaling it.

Theorem 6. Consider a twisted Edwards curve defined over $\mathbb{F}_{p}$ given by equation ax ${ }^{2}+y^{2}=1+$ $d x^{2} y^{2}$. If $-a$ is a square in $\mathbb{F}_{p}$, then the map $(x, y) \rightarrow(x / \sqrt{-a}, y)$ defines the curve $-x^{2}+y^{2}=$ $1+(-d / a) x^{2} y^{2}$. We denote by $f=\sqrt{-a}$ the scaling factor. 
Proof. The result follows directly from the map's definition.

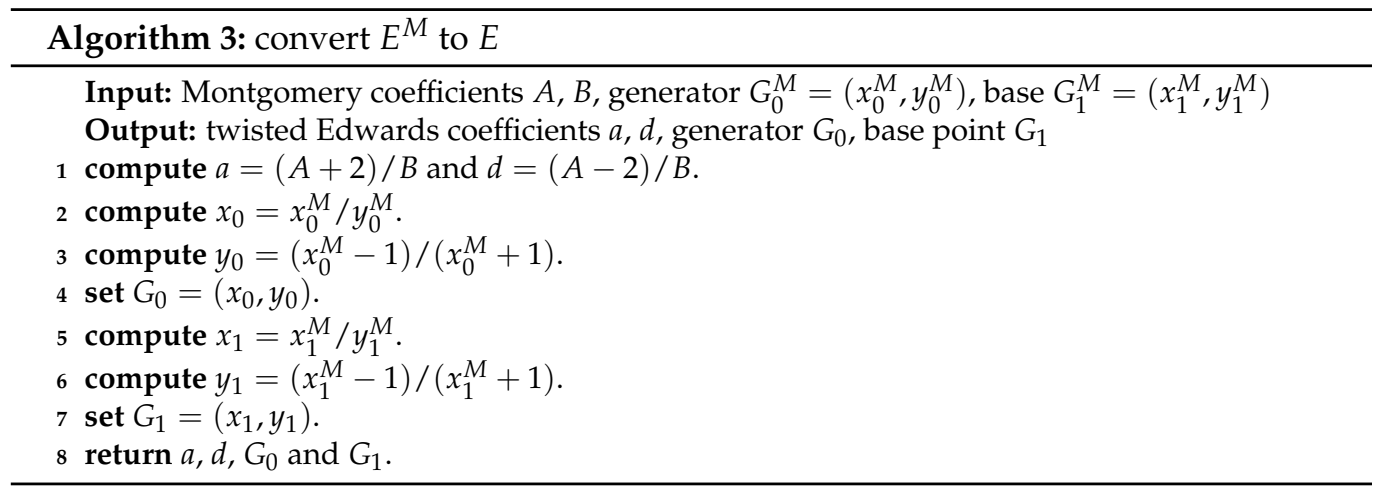

The following Algorithm 4 rescales, if possible, the twisted Edwards curve found in the previous step as described in the previous theorem. It also converts the generator and base points to the new coordinates. After applying the algorithm, the map that transforms $E^{M}$ to $E$ becomes the composition of maps from Theorems 5 and 6 .

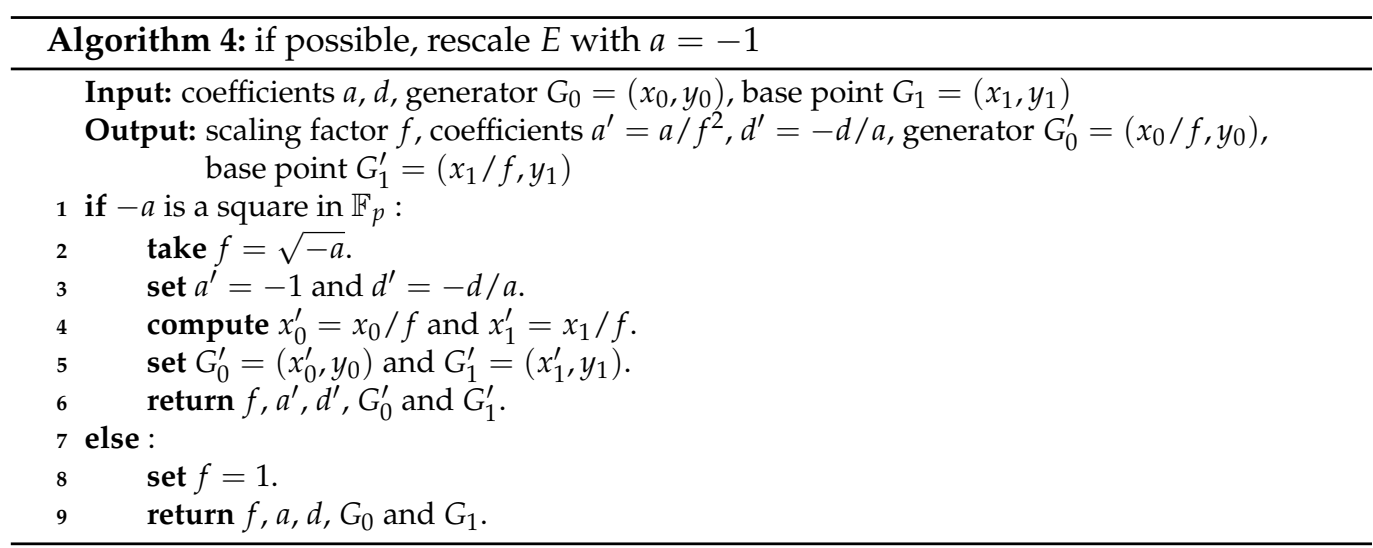

\section{Security Analysis}

This section specifies the safety criteria that the elliptic curve should satisfy. The choices of security parameters are based on the joint work of Bernstein and Lange summarized in [18]. In Appendix A, we provide an implementation of the algorithm that should be run after finding the elliptic curve as proposed in the previous section. The algorithm is based on the code from [42], which is an extension of the original SAGE code from [18], to general twisted Edwards curves.

Curve Parameters: We check that all given parameters describe a well-defined elliptic curve over a prime finite field:

- $\quad$ The given number $p$ is prime;

- The given parameters define an equation that corresponds to an elliptic curve;

- The product of $h$ and $l$ results into the order of the curve and the point $G_{0}$ is a generator;

- The given number $l$ is prime and the point $G_{1}$ is a base point.

Elliptic Curve Discrete Logarithm Problem: We check that the discrete logarithm problem remains difficult in the given curve. For that, we check it is resistant to the following known attacks:

- $\quad$ Rho method (Section V.1, [17]): we require the cost for the rho method, which takes on average around $0.886 \sqrt{l}$ additions, to be above $2^{100}$;

- Additive and multiplicative transfers (Section V.2, [17]): we require the embedding degree to be at least $(l-1) / 100$; 
- High discriminant (Section IX.3, [17]): we require the complex-multiplication field discriminant $D$ to be larger than $2^{100}$.

Elliptic Curve Cryptography: We check if the curve is suitable for ECC:

- Ladders [16]: check the curve supports the Montgomery ladder;

- Twists (twist, [18]): check if it is secure against the small-subgroup attack, invalidcurve attacks and twisted-attacks;

- Completeness (complete, [18]): check if the curve has complete single-scalar and multiple-scalar formulas. It is enough to check that there is only one point of order 2 and 2 of order 4;

- Indistinguishability [43]: check availability of maps that turn elliptic-curve points indistinguishable from uniform random strings.

\section{Implementation}

Ethereum, the second-largest blockchain, uses BN256 to generate and verify ZKSNARK proofs. BN256 is a pairing-friendly elliptic curve of prime order

\section{$p=21888242871839275222246405745257275088548364400416034343698204186575808495617$.}

In order to prove ECC statements with ZK-SNARKs, Ethereum needed a new curve defined over $\mathbb{F}_{p}$. In this section, we present a SAGE implementation of the algorithms presented in the previous sections, and we use them to generate Baby Jubjub, a twisted Edwards elliptic curve suitable for ZK-SNARK circuits in Ethereum.

First, we implemented Algorithm 1 in Listing 1, which generates a Montgomery curve $E^{M}$ with the smallest $A$ satisfying the conditions we described in Section 4.2. In the last lines of code, we instantiate the functions using the prime number $p$, which is the order of BN256 curve, and enforcing the resulting curve to have cofactor $h=8$.

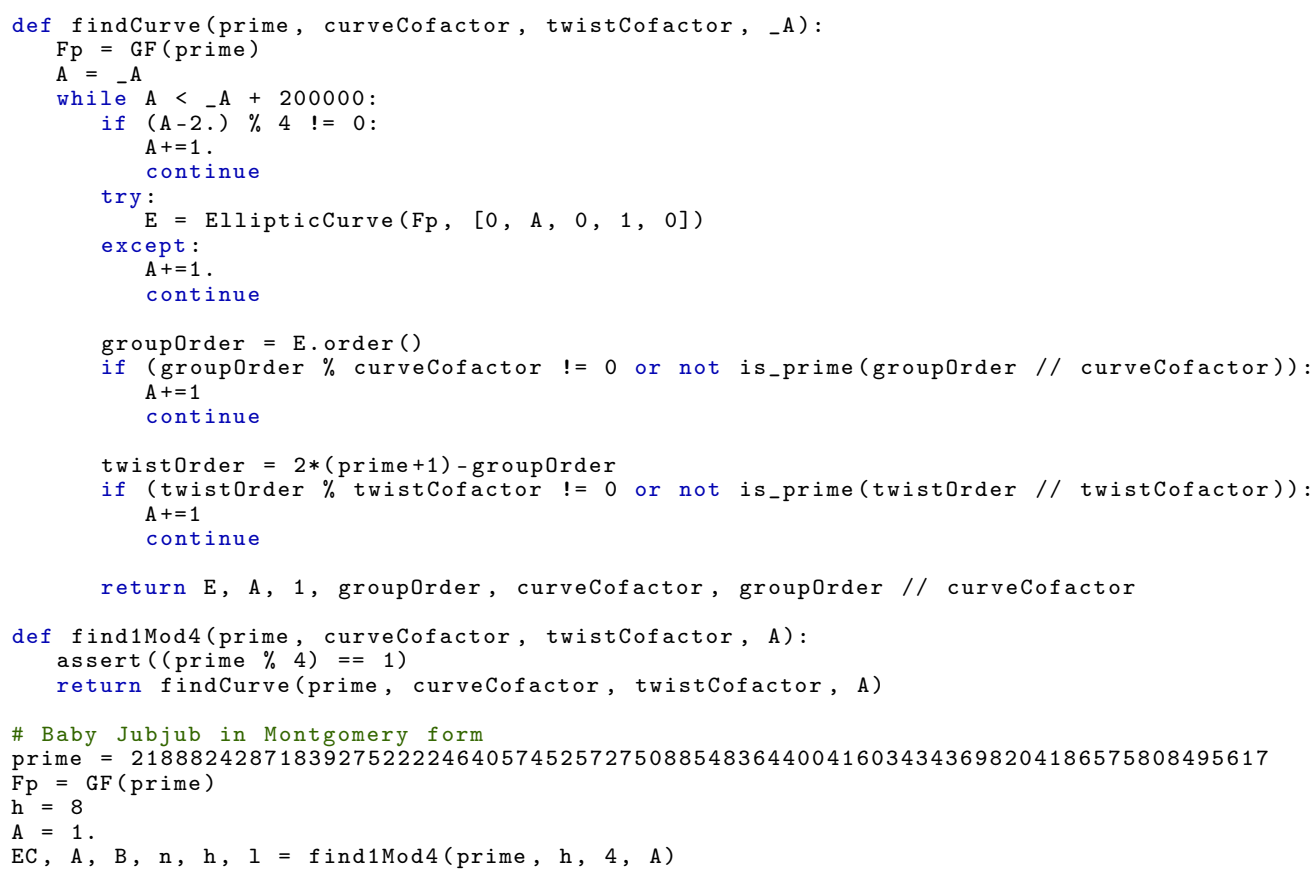

Listing 1. Generation of $E^{M}$.

The result from Listing 1 is that the smallest A satisfying our conditions is $A=168,698$. As a result, the Montgomery form of Baby Jubjub is defined over $\mathbb{F}_{p}$ by equation:

$$
y^{2}=x^{3}+168,698 x^{2}+x .
$$

The function findCurve also returns the order of the curve, which in this case is 


$$
n=21888242871839275222246405745257275088614511777268538073601725287587578984328,
$$

where $n=h \times l$, with $h=8$, and $l$ is the large prime number

$$
l=2736030358979909402780800718157159386076813972158567259200215660948447373041 .
$$

The next step is to generate a generator and a base point for Baby Jubjub in Montgomery form. For that, we implemented Algorithm 2 in Listing 2. Recall that the algorithm is deterministic and takes as a generator $G_{0}^{M}$, the point of the curve of order $n$ with smallest $x$-coefficient, and as a base point, $G_{1}^{M}=h \cdot G_{0}^{M}$. The last two lines of code are used to find a valid generator and base point for Baby Jubjub in Montgomery form.

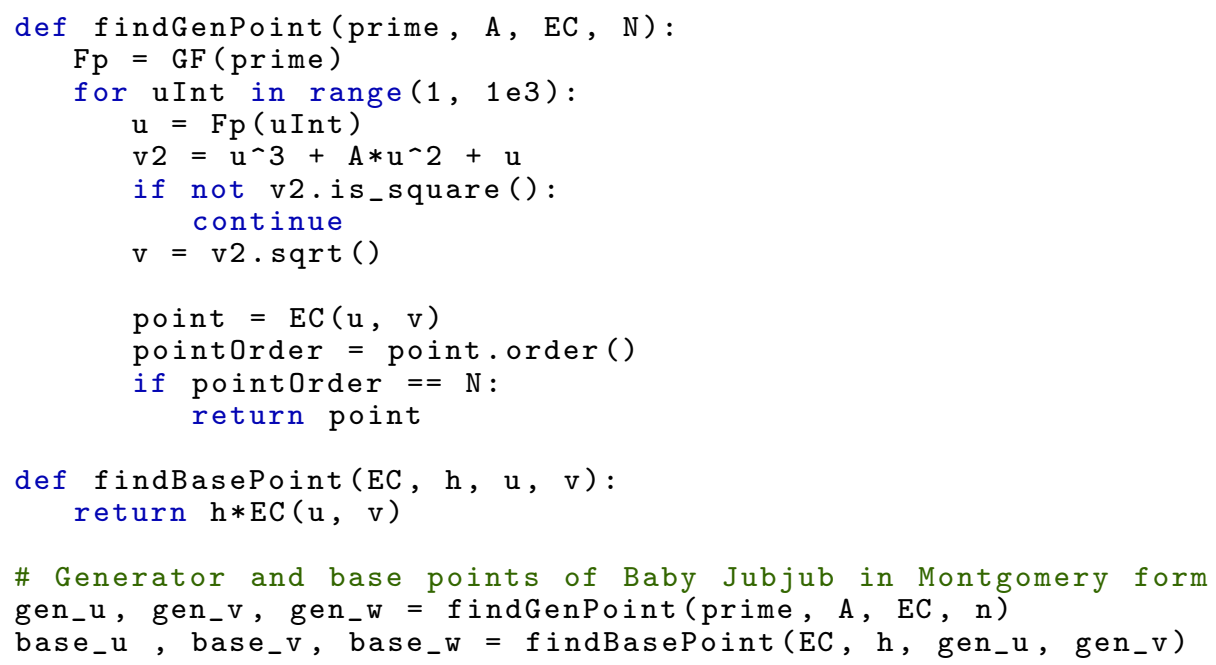

Listing 2. Generator $G_{0}$ and base point $G_{1}$ of $E^{M}$.

The resulting points from Listing 2 are the generator $G_{0}^{M}=\left(x_{0}^{M}, y_{0}^{M}\right)$ with coordinates

$x_{0}^{M}=7$,

$y_{0}^{M}=4258727773875940690362607550498304598101071202821725296872974770776423442226$,

and the base point $G_{1}^{M}=\left(x_{1}^{M}, y_{1}^{M}\right)$ with coordinates

$x_{1}^{M}=7117928050407583618111176421555214756675765419608405867398403713213306743542$,

$y_{1}^{M}=14577268218881899420966779687690205425227431577728659819975198491127179315626$.

Algorithm 3 maps a Montgomery curve to its twisted Edwards form. We divided the algorithm in three different functions. The first function mont_to_ted converts a Montgomery point to a twisted Edwards point, the function ted_to_mont does the opposite, and is_on_ted checks if a point is a solution to a given twisted Edwards equation. Although the last two functions are not needed in the original algorithm, we implemented them in order to have sanity checks after the conversion from Montgomery to twisted Edwards form.

After the conversion maps, we get that the twisted Edwards form of Baby Jubjub is described by equation

$$
168700 x^{2}+y^{2}=1+168696 x^{2} y^{2} .
$$

The code from Listing 3 also outputs the generator $G_{0}=\left(x_{0}, y_{0}\right)$ and base point $G_{1}=\left(x_{1}, y_{1}\right)$ in twisted Edwards form. The specific outputs are that $G_{0}$ has coordinates 


$$
\begin{aligned}
& x_{0}=995203441582195749578291179787384436505546430278305826713579947235728471134, \\
& y_{0}=5472060717959818805561601436314318772137091100104008585924551046643952123905, \\
& \text { and } G_{1} \text { has coordinates } \\
& x_{1}=5299619240641551281634865583518297030282874472190772894086521144482721001553 \\
& y_{1}=16950150798460657717958625567821834550301663161624707787222815936182638968203 .
\end{aligned}
$$

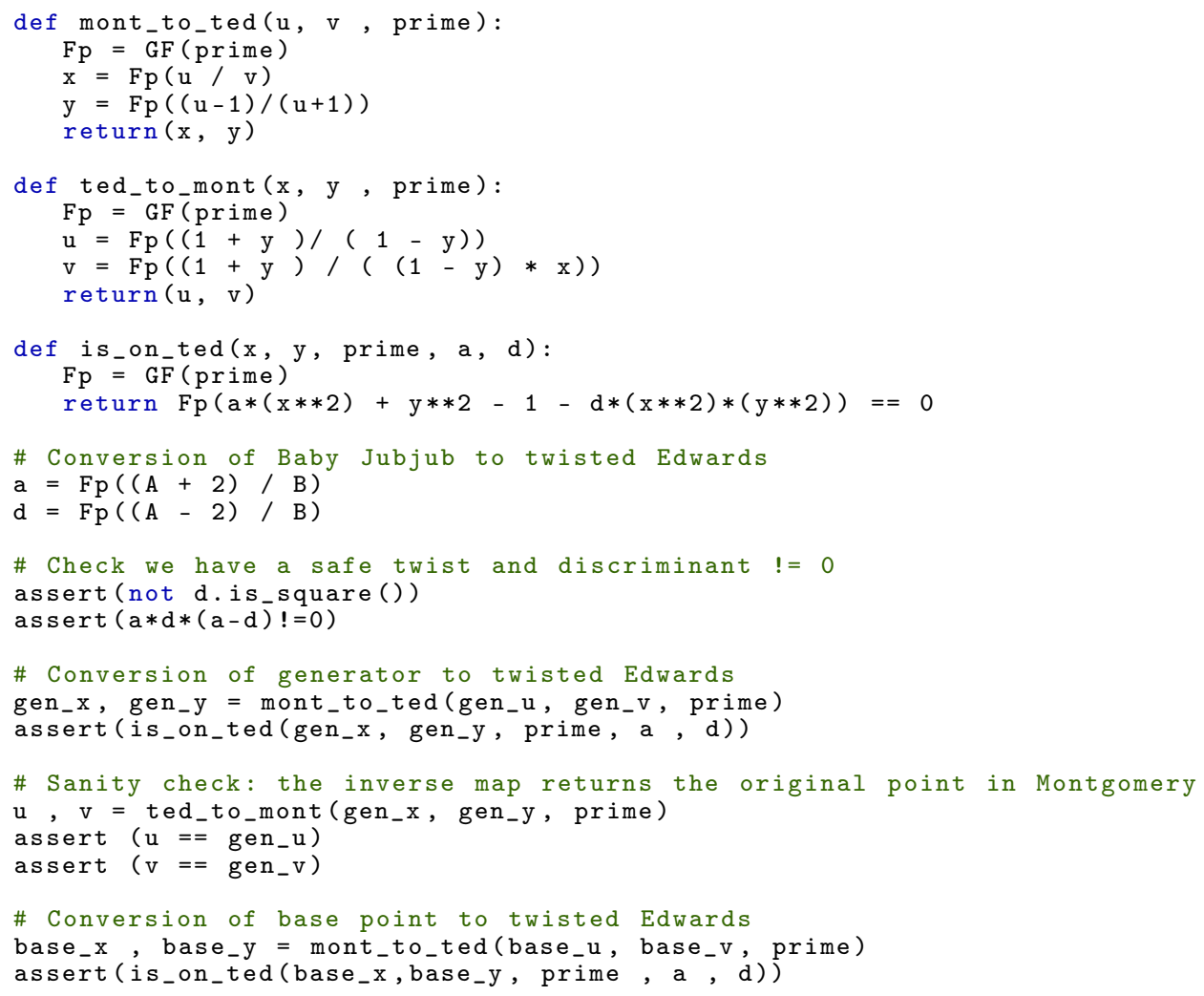

Listing 3. Conversion of $E^{M}$ to $E$.

The last Algorithm 4 tries to escalate the twisted Edwards form of the curve, so that the equation has parameter $a=-1$. This last step is implemented in Listing 4 and in the case of Baby Jubjub, the resulting scaling factor is

\section{$f=1911982854305225074381251344103329931637610209014896889891168275855466657090$.}

This way, the optimal version of Baby Jubjub in twisted Edwards form is given by equation

$$
-x^{2}+y^{2}=1+d^{\prime} x^{2} y^{2}
$$

where

$d^{\prime}=12181644023421730124874158521699555681764249180949974110617291017600649128846$. 


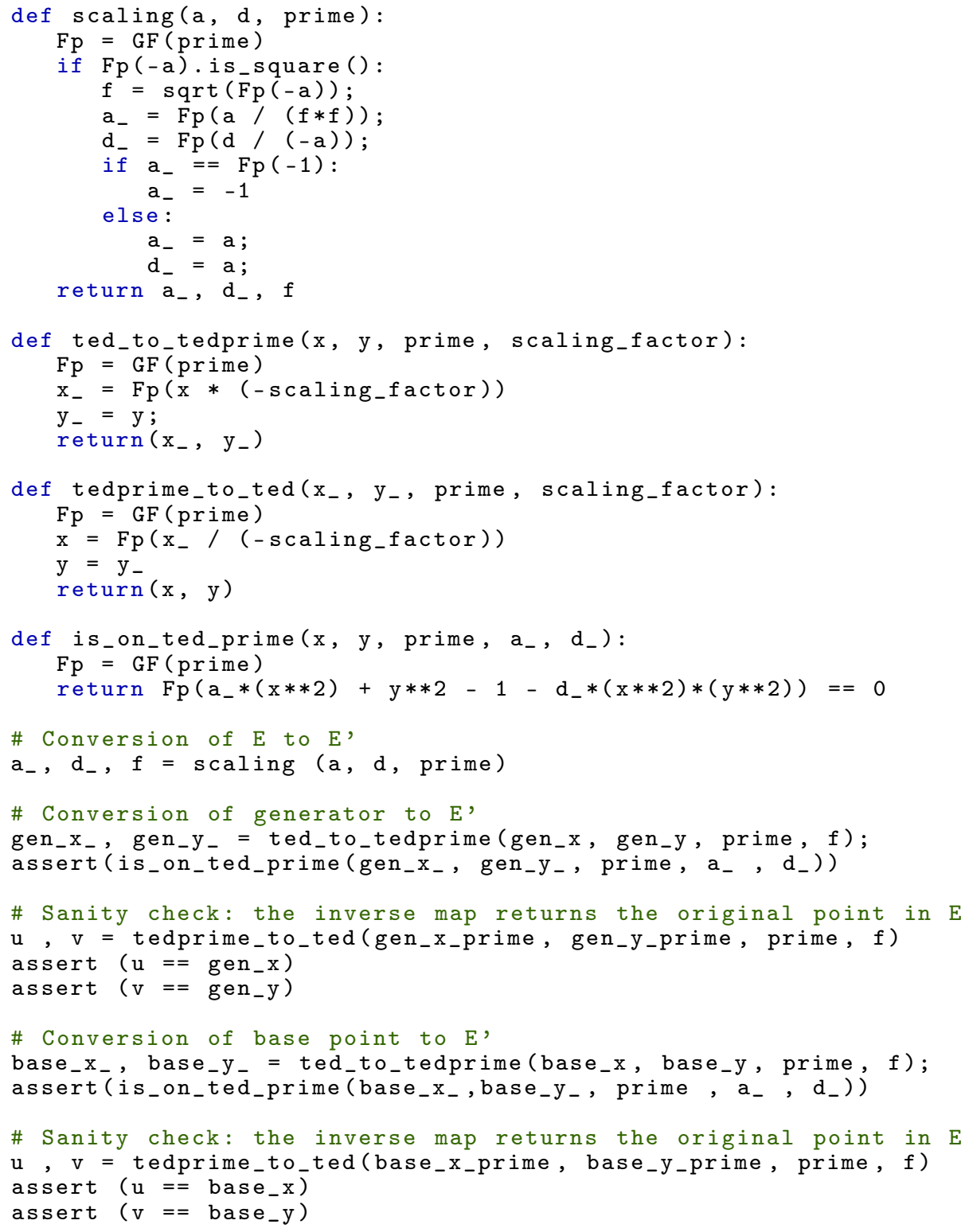

Listing 4. Scaling of $E$ to $E^{\prime}$.

After generating Baby Jubjub, we checked that the curve passed all safety checks described in Section 5. The security evidence is shown in [44]. The determinism and transparency of the procedure allows any party to reproduce the generation of the curve and ensure its resilience against best-known security attacks [45].

Baby Jubjub curve was accepted as an Ethereum Improvement Proposal [11], and it is currently being used in several projects running over Ethereum, such as Hermez, a Layer-2 payment system, and Tornado Cash, a payment mixer.

\section{Discussion}

In the recent years, ZK proofs arose as a potential solution to blockchain privacy and scalability issues and, today, we can see many zero-knowledge protocols integrated and deployed in various blockchain projects. The use of cryptography in the blockchain space arises new efficiency needs that motivate novel lines of research that combine theoretical and practical aspects. In particular, the recent implementation of ZK protocols has had a huge impact in the interest for generating types of curves with special properties. The correct and transparent generation of new elliptic curves is paramount to the 
success of cryptographic primitives that can help blockchains improve their privacy and scalability guarantees.

In this paper, we presented a deterministic algorithm for generating twisted Edwards elliptic curves defined over a given prime field. We also provided an algorithm for checking the safety of a curve against best known security attacks. Additionally, we gave an example that puts theory into practice: we detailed the generation of the twisted Edwards curve Baby Jubjub, which is currently being deployed and used in several projects based on Ethereum blockchain. The generation of other types of curves, such as pairing-friendly and prime order curves and efficient pairs of cyclic curves, which can help build more efficient ZK-SNARK schemes and opens the door to the creation of succinct blockchains, remains as future work.

Author Contributions: Conceptualization, M.B.-M. and B.W.; methodology, M.B.-M. and B.W.; software, B.W.; validation, M.B.-M.; formal analysis, M.B.-M.; investigation, B.W.; resources, B.W. and J.B.; writing—original draft preparation, M.B.-M.; writing—review and editing, M.B.-M., V.D. and J.L.M.-T.; supervision, V.D. and J.L.M.-T.; project administration, J.B., V.D. and J.L.M.-T.; funding acquisition, J.B. and V.D. All authors have read and agreed to the published version of the manuscript.

Funding: This research has been partially funded by the projects Project RTI2018-102112-B-100 (AEI/FEDER, UE), i3Market (H2020-ICT-2019-2 grant number 871754) and TCO-RISEBLOCK (PID2019110224RB-I00).

Institutional Review Board Statement: Not applicable.

Informed Consent Statement: Not applicable.

Data Availability Statement: Not applicable.

Conflicts of Interest: The authors declare no conflicts of interest.

\section{Abbreviations}

The following abbreviations are used in this manuscript:

$\begin{array}{ll}\text { BN } & \text { Barreto-Naehrig } \\ \text { BLS } & \text { Barreto-Lynn-Scott } \\ \text { CCPA } & \text { California Consumer Privacy Act } \\ \text { ECC } & \text { Elliptic-Curve Cryptography } \\ \text { EdDSA } & \text { Edwards Digital Signature Algorithm } \\ \text { EVM } & \text { Ethereum Virtual Machine } \\ \text { GDPR } & \text { General Data Protection Regulation } \\ \text { IRTF } & \text { Internet Research Task Force } \\ \text { MNT } & \text { Miyaji-Nakabayashi-Takano } \\ \text { NP } & \text { Nondeterministic Polynomial Time } \\ \text { ZK } & \text { Zero-Knowledge } \\ \text { ZK-SNARK } & \text { Zero-Knowledge Succinct Non-interactive Argument of Knowledge }\end{array}$

\section{List of Mathematical Symbols}

The following mathematical symbols are used in this manuscript:

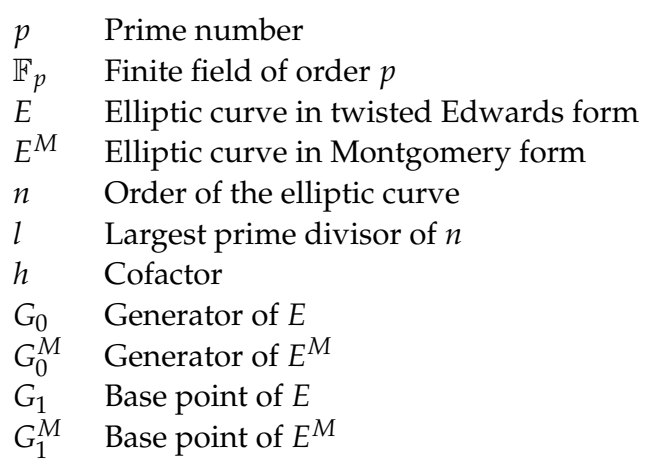




\section{Appendix A. Safety Checks against Known Attacks}

We provide an implementation of an algorithm based on the code from [42], that checks if a given twisted Edwards curve is safe against the attacks described in Section 5.

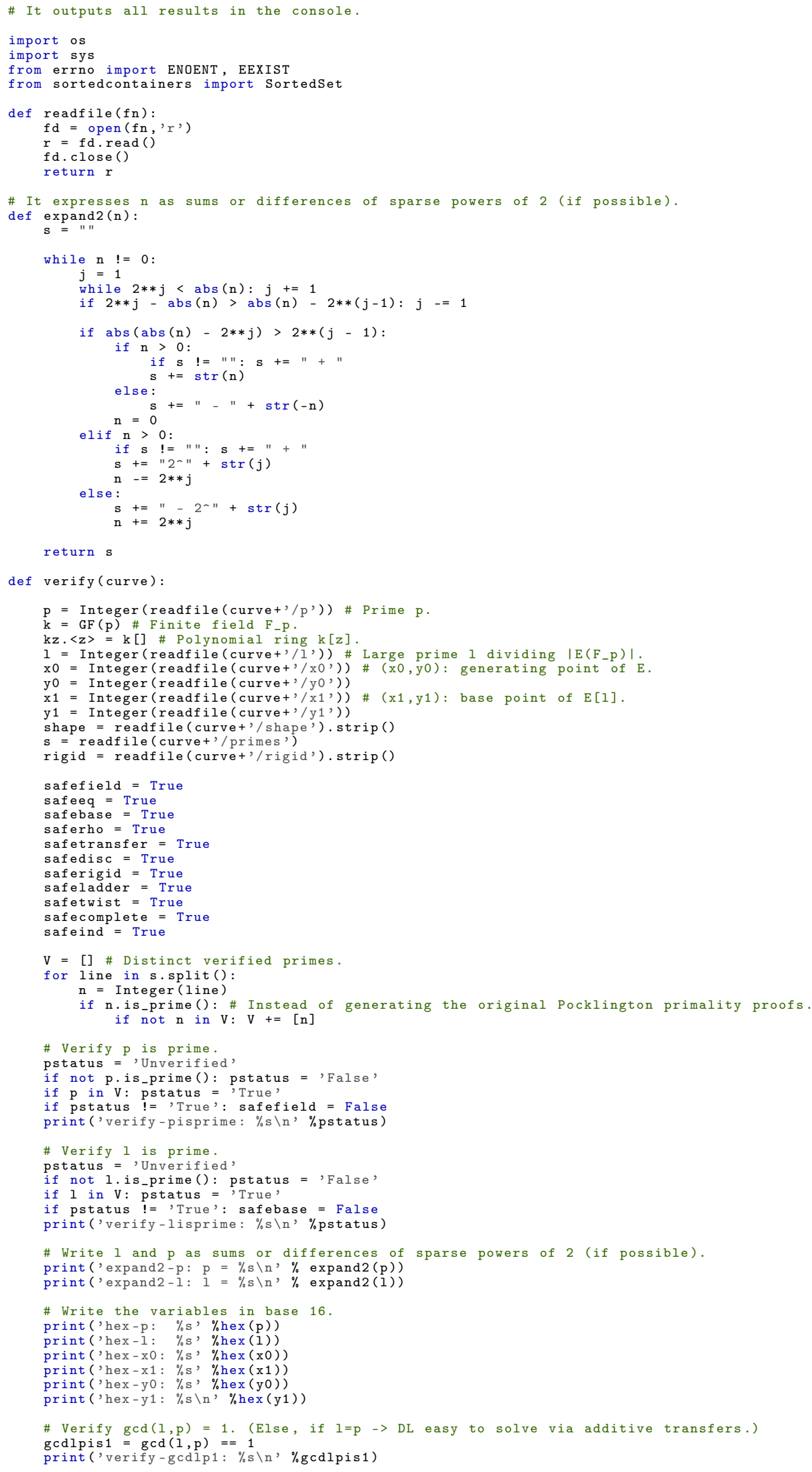




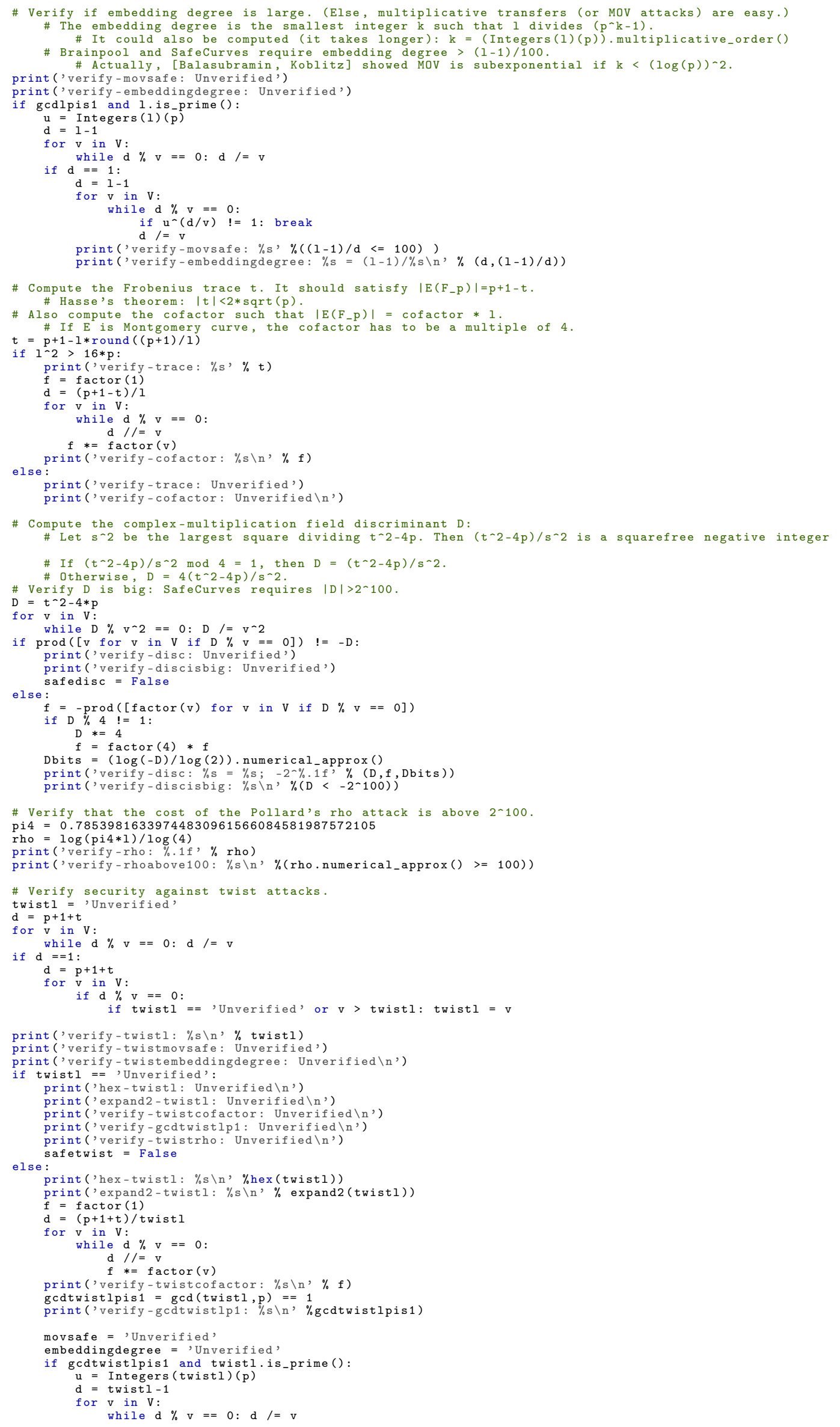




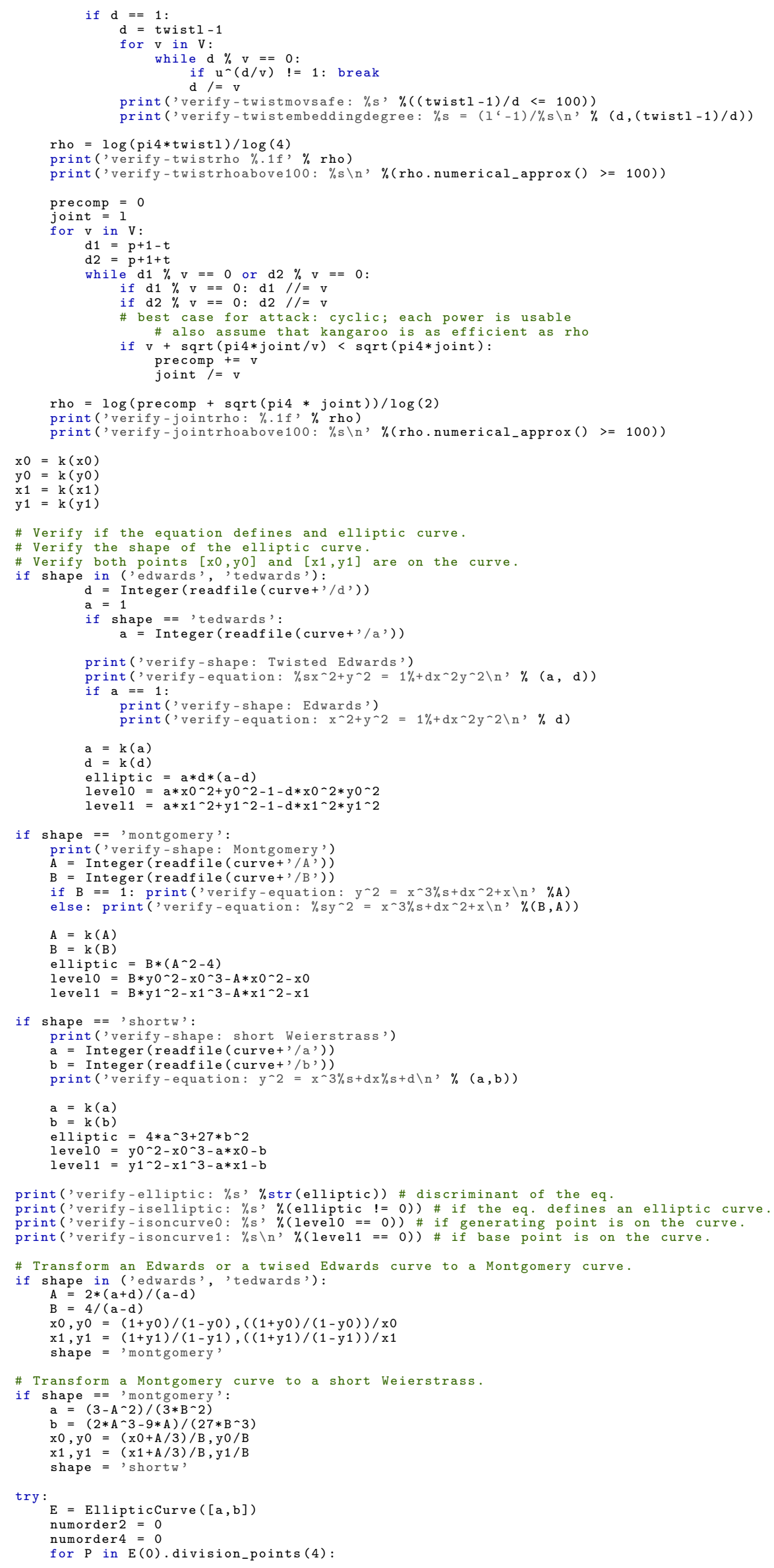




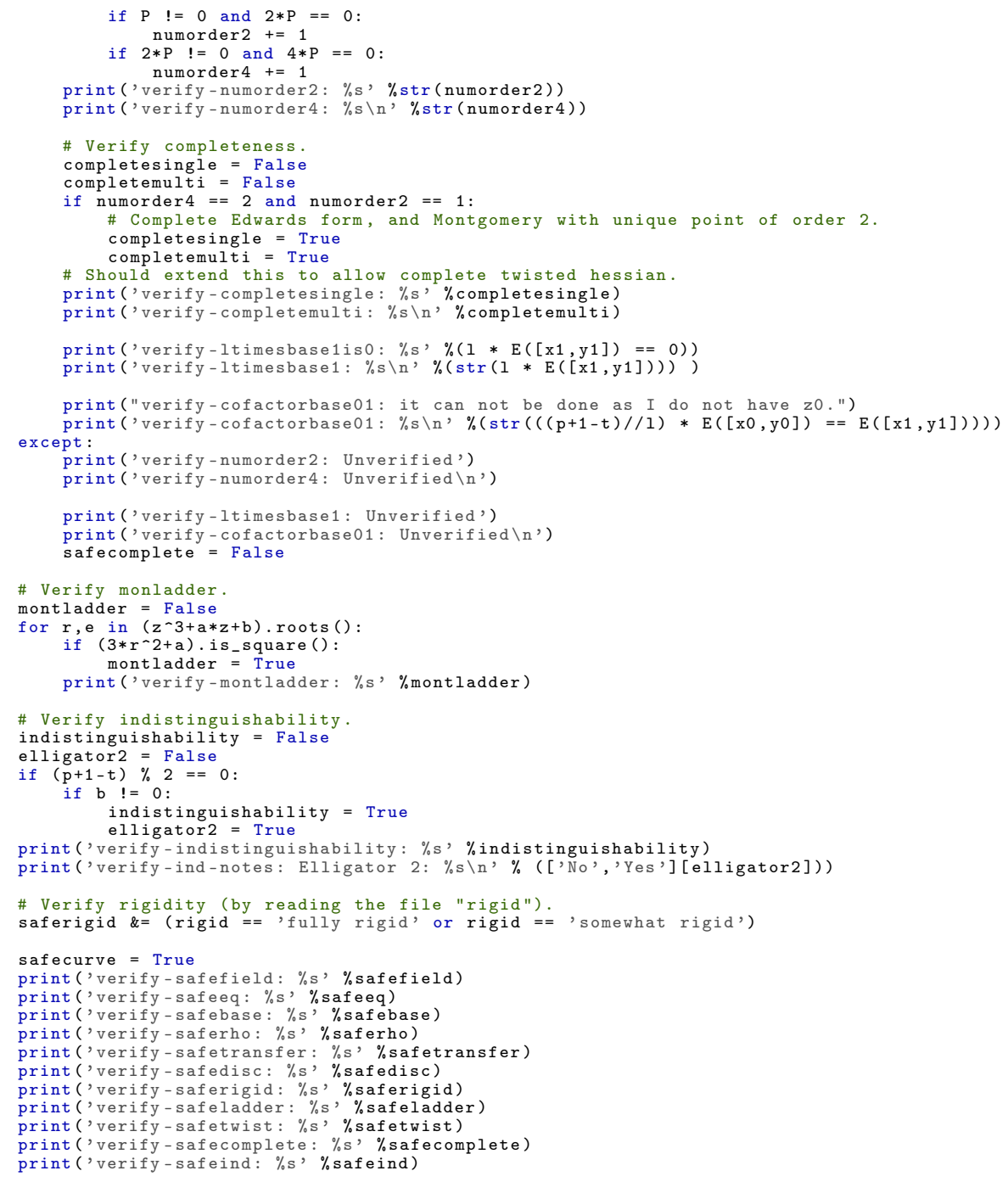

Listing A1. Algorithm written in SAGE that verifies if a given twisted Edwards curve passes the checks described in Section 5.

\section{References}

1. Koblitz, N. Elliptic Curve Cryptosystems. Math. Comput. 1987, 48, 203-209. [CrossRef]

2. Miller, V.S. Use of Elliptic Curves in Cryptography. In Advances in Cryptology-CRYPTO '85 Proceedings, Proceedings of the Annual International Cryptology Conference, Santa Barbara, CA, USA, 18-22 August 1985; Williams, H.C., Ed.; Springer: Berlin/Heidelberg, Germany, 1986; pp. 417-426. [CrossRef]

3. Hankerson, D.; Menezes, A.J.; Vanstone, S. Guide to Elliptic Curve Cryptography; Springer: Berlin, Heidelberg, Germany, 2003.

4. Nakamoto, S. Bitcoin: A Peer-to-Peer Electronic Cash System. 2009. Available online: http://www.bitcoin.org/bitcoin.pdf (accessed on 30 October 2021)

5. Noether, S.; Mackenzie, A.; Lab, T. Ring Confidential Transactions. Ledger 2016, 1, 1-18. [CrossRef]

6. $\quad$ Ben-Sasson, E.; Chiesa, A.; Garman, C.; Green, M.; Miers, I.; Tromer, E.; Virza, M. Zerocash: Decentralized Anonymous Payments from Bitcoin. In Proceedings of the IEEE Symposium on Security and Privacy, Berkeley, CA, USA, 18-21 May 2014; IEEE Computer Society: Los Alamitos, CA, USA, 2014, pp. 459-474. [CrossRef]

7. Goldwasser, S.; Micali, S.; Rackoff, C. The knowledge complexity of interactive proof systems. SIAM J. Comput. 1989, 18, 186-208. [CrossRef]

8. Parno, B.; Howell, J.; Gentry, C.; Raykova, M. Pinocchio: Nearly Practical Verifiable Computation. Commun. ACM 2016, 59, 103-112. [CrossRef]

9. Groth, J. On the Size of Pairing-Based Non-interactive Arguments. In Advances in Cryptology—EUROCRYPT 2016; Springer: Berlin/Heidelberg, Germany, 2016; pp. 305-326. [CrossRef] 
10. Gabizon, A.; Williamson, Z.J.; Ciobotaru, O. PLONK: Permutations over Lagrange-Bases for Oecumenical Noninteractive Arguments of Knowledge. Cryptology ePrint Archive, Report 2019/953. 2019. Available online: https://ia.cr/2019/953 (accessed on 30 October 2021).

11. WhiteHat, B.; Bellés, M.; Baylina, J. Baby Jubjub Elliptic Curve. Ethereum Improvement Proposal, EIP-2494. 29 January 2020. Available online: https://github.com/ethereum/EIPs/blob/master/EIPS/eip-2494.md (accessed on 30 October 2021).

12. Electric Coin Company. What Is Jubjub? Available online: https://z.cash/technology/jubjub/ (accessed on 30 October 2021).

13. Libert, B.; Mouhartem, F.; Stehlé, D. Notes from the Master Course Cryptology and Security at the École Normale Supérieure de Lyon. Tutorial 8, 2016-2017. Available online: https://fmouhart.epheme.re/Crypto-1617/TD08.pdf (accessed on 30 October 2021).

14. Josefsson, S.; Liusvaara, I. Edwards-Curve Digital Signature Algorithm (EdDSA). Internet Research Task Force (IRTF). Request for Comments: 8032, January 2017. Available online: https://tools.ietf.org/html/8032 (accessed on 30 October 2021),

15. Bernstein, D.J.; Birkner, P.; Joye, M.; Lange, T.; Peters, C. Twisted Edwards Curves. In Progress in Cryptology-AFRICACRYPT 2008; Vaudenay, S., Ed.; Springer: Berlin/Heidelberg, Germany, 2008; pp. 389-405. [CrossRef]

16. Montgomery, P.L. Speeding the Pollard and Elliptic Curve Methods of Factorization. Math. Comput. 1987, 48, 243-243. [CrossRef]

17. Blake, I.; Seroussi, G.; Smart, N. Elliptic Curves in Cryptography; London Mathematical Society Lecture Note Series; Cambridge University Press: Cambridge, UK, 1999; Volume 256.

18. Bernstein, D.J.; Lange, T. SafeCurves: Choosing Safe Curves for Elliptic-Curve Cryptography. Available online: https: / / safecurves.cr.yp.to (accessed on 30 October 2021).

19. European Parliament and Council of the European Union. Regulation (EU) 2016/679 of the EUROPEAN PARLIAMENT and of the Council of 27 April 2016 on the Protection of Natural Persons with Regard to the Processing of Personal Data and on the Free Movement of Such Data, and Repealing Directive 95/46/EC (General Data Protection Regulation). 2016. Available online: https: / / eur-lex.europa.eu/eli/reg/2016/679 (accessed on 30 October 2021).

20. The California Consumer Privacy Act of 2018. An Act to Add Title 1.81.5 (Commencing with Section 1798.100) to Part 4 of Division 3 of the Civil Code, Relating to privacy, 2018. Available online: https:/ /leginfo.legislature.ca.gov/faces/codes_displayText.xhtml? division $=3$. \& part $=4$. \&lawCode $=$ CIV \&title $=1.81 .5$ (accessed on 30 October 2021).

21. Bellés-Muñoz, M.; Baylina, J.; Daza, V.; Muñoz, J.L. New Privacy Practices for Blockchain Software. IEEE Softw. 2021, [CrossRef]

22. Salleras, X.; Daza, V. ZPiE: Zero-Knowledge Proofs in Embedded Systems. Mathematics 2021, 9, 2569. [CrossRef]

23. Sestrem Ochôa, I.; Reis Quietinho Leithardt, V.; Calbusch, L.; De Paz Santana, J.F.; Delcio Parreira, W.; Oriel Seman, L.; Albenes Zeferino, C. Performance and Security Evaluation on a Blockchain Architecture for License Plate Recognition Systems. Appl. Sci. 2021, 11, 1255. [CrossRef]

24. Barreto, P.S.L.M.; Naehrig, M. Pairing-Friendly Elliptic Curves of Prime Order. In Proceedings of the 12th International Conference on Selected Areas in Cryptography (SAC'05), Kingston, ON, Canada, 11-12 August 2005; Springer: Berlin/Heidelberg, Germany, 2005; p. 319-331. [CrossRef]

25. Barreto, P.S.L.M.; Lynn, B.; Scott, M. Constructing Elliptic Curves with Prescribed Embedding Degrees. In Security in Communication Networks; Springer: Berlin/Heidelberg, Germany, 2003; pp. 257-267. [CrossRef]

26. Kasamatsu, K.; Kanno, S.; Kobayashi, T.; Kawahara, Y. Barreto-Naehrig Curves. Network Working Group. Internet-Draft. February 2014. Available online: https://tools.ietf.org/html/draft-kasamatsu-bncurves-01 (accessed on 30 October 2021).

27. Yonezawa, S.; Chikara, S.; Kobayashi, T.; Saito, T. Pairing-Friendly Curves. Network Working Group. Internet-Draft. January 2019. Available online: https://tools.ietf.org/html/draft-yonezawa-pairing-friendly-curves-00 (accessed on 30 October 2021).

28. Masson, S.; Sanso, A.; Zhang, Z. Bandersnatch: A Fast Elliptic Curve Built over the BLS12-381 Scalar Field. Cryptology ePrint Archive, Report 2021/1152. 2021. Available online: https://ia.cr/2021/1152 (accessed on 30 October 2021).

29. Ben-Sasson, E.; Chiesa, A.; Tromer, E.; Virza, M. Scalable Zero Knowledge via Cycles of Elliptic Curves. In Advances in Cryptology_CRYPTO 2014; Garay, J.A.; Gennaro, R., Eds.; Springer: Berlin/Heidelberg, Germay, 2014; pp. 276-294. [CrossRef]

30. Bowe, S.; Chiesa, A.; Green, M.; Miers, I.; Mishra, P.; Wu, H. ZEXE: Enabling Decentralized Private Computation. In Proceedings of the 2020 IEEE Symposium on Security and Privacy (SP), San Francisco, CA, USA, 18-21 May 2020; IEEE Computer Society: Los Alamitos, CA, USA, 2020; pp. 947-964. [CrossRef]

31. Housni, Y.E.; Guillevic, A. Optimized and Secure Pairing-Friendly Elliptic Curves Suitable for One Layer Proof Composition. Cryptology ePrint Archive, Report 2020/351. 2020. Available online: https:/ /ia.cr/2020/351 (accessed on 30 October 2021).

32. Miyaji, A.; Nakabayashi, M.; Nonmembers, S. New explicit conditions of elliptic curve traces for FR-reduction. IEICE Trans. Fundam. Electron. Commun. Comput. Sci. 2001, 84, 1234-1243.

33. Koblitz, N. A Course in Number Theory and Cryptography, 2nd ed.; Graduate Texts in Mathematics; Springer: Berlin/Heidelberg, Germany, 1994.

34. Silverman, J.H. The Arithmetic of Elliptic Curves. Graduate Texts in Mathematics; Springer: New York, NY, USA, $1994 ;$ Volume 106.

35. Washington, L.C. Elliptic Curves. Number theory and Cryptography, 2nd ed.; Chapman \& Hall/CRC Press: Boca Raton, FL, USA, 2008.

36. Okeya, K.; Kurumatani, H.; Sakurai, K. Elliptic Curves with the Montgomery-Form and Their Cryptographic Applications. In Proceedings of the Third International Workshop on Practice and Theory in Public Key Cryptography: Public Key Cryptography (PKC '00), Melbourne, VIC, Australia, 18-20 January 2000; Springer: London, UK, 2000; pp. 238-257. [CrossRef] 
37. Bernstein, D.J.; Lange, T. Faster Addition and Doubling on Elliptic Curves. In Advances in Cryptology—ASIACRYPT 2007; Springer: Berlin/Heidelberg, Germany, 2007; pp. 29-50. [CrossRef]

38. Hisil, H.; Wong, K.K.H.; Carter, G.; Dawson, E. Twisted Edwards Curves Revisited. In Advances in Cryptology—ASIACRYPT 2008; Springer: Berlin/Heidelberg, Germany, 2008; pp. 326-343. [CrossRef]

39. Langley, A.; Hamburg, M.; Turner, S. Elliptic Curves for Security. Internet Research Task Force (IRTF). Request for Comments: 7748. January 2016. Available online: https://tools.ietf.org/html/7748 (accessed on 30 October 2021),

40. Bowe, S. Derivation of Jubjub Elliptic Curve. GitHub. 2019. Available online: https://github.com/zkcrypto/jubjub/blob/ master/doc/derive/derive.sage (accessed on 30 October 2021).

41. Bernstein, D.J.; Lange, T. Montgomery curves and the Montgomery Ladder. Cryptology ePrint Archive, Report 2017/293. 2017. Available online: https: / ia.cr/2017/293 (accessed on 30 October 2021).

42. Hopwood, D. Supporting Evidence for Security of the Jubjub Curve to Be Used in Zcash. Available online: https://github.com/ daira/jubjub/blob/master/verify.sage (accessed on 30 October 2021).

43. Bernstein, D.J.; Hamburg, M.; Krasnova, A.; Lange, T. Elligator: Elliptic-Curve Points Indistinguishable from Uniform Random Strings. In Proceedings of the 2013 ACM SIGSAC Conference on Computer and Communications Security (CCS '13), Berlin Germany, 4-8 November 2013; Association for Computing Machinery: New York, NY, USA, 2013; pp. 967-980. [CrossRef]

44. WhiteHat, B. Baby Jubjub Supporting Evidence. GitHub. 2018. Available online: https://github.com/barryWhiteHat/baby_ jubjub (accessed on 30 October 2021).

45. Singh, S.K.; Tanwar, S. Analysis of Software Testing Techniques: Theory to Practical Approach. Indian J. Sci. Technol. 2016, 9, 1-6. [CrossRef] 